\title{
DEVELOPMENT OF FIBER OPTICS SPECTROSCOPIC TOOTH COLORIMETER USING WHITE LED
}

\author{
Sheng-Hong Yu', Cheng-Hau Chen ${ }^{2}$, Hulhua Kenny Chiang' \\ 'Institute of Biomedical Engineering, National Yang-Ming University, Taipei \\ ${ }^{2}$ Institute of Biophotonics Engineering, National Yang-Ming University, Taipei, \\ Taiwan
}

\begin{abstract}
Tooth colorimeter provides the determination of patient's tooth shade, and assists dentists, technicians and patients in satisfying the clinical needs for dental medicine. The traditional visual matching approaches of tooth shade tabs had some limitations that are based on subjective color perception and could be easily affected by environmental factors. In this research, we have developed a fiber optics spectroscopic tooth colorimeter with white LED, instead of the tungsten lamp to improve the size and heat problems. We have also developed a Whiteness-Yellowness index to simplify the tooth color information and have achieved an objectively and quantitatively tooth color representation.
\end{abstract}

Biomed Eng Appl Basis Comm, 2004 (April); 16: 73-78.

\section{INTRODUCTION}

Color is a visible perception and dependent on three elements: (1) illumination (spectrum energy distribution); (2) absorbance and scattering of illuminated object; (3) observer (spectral response related to wavelength) [1]. It's very difficult to detemine the color of some objects accurately, such as human teeth that have many undesirable properties, for cxample, the surface roughness and inhomogeneous of intrinsic materials among other persons. In clinical works, the tooth color determination was mainly affected by the environmental factors, for instance, the interference of indoor lightness. However, the variation of the color perception of dentists is easily ignored [2].

Received: Dec 18, 2003; Accepted: Jan 29, 2004

Correspondence: Huihua Kenny Chiang, Professor institute of Biomedical Engineering.

National Yang-Ming University, No.155, Section 2, Li-Nung Street, Taipei, 112, Taiwan

E-mail: hkchiang@ym.edu.tw
It is very important to match the teeth color more objectively and accurately in dental clinical works, and to enable the effective and consistent communication with dental technicians. In addition, dental laboratories admit to a 6 percent remake rate annually. Half of these remakes ( 3 percent) occur for misinterpretation and failure to match shades accurately [3]. Consequently in clinics, the needs for more reliable and reproducible determination of teeth color has become more significantly.

In this study, we have developed a tooth colorimeter system, based on the spectroscopic mechanism. This system consists of a minispectrometer for spectrum recording, a set of white LED for illumination, a speciaily designed optical fiber cable for light transmission, and a self-developed analysis algorithm for determining the tooth colors. We have successfulty implemented this system for teeth color determination on a set of tooth phantom consisting of 26 teeth of different color coordinates. 


\section{PRINCIPLES OF COLORIMETRY}

CIE (International Commission on Illumination) specified two color standards that are the most widely used in color estimation, the CIEXYZ (CIE 1931) and CIELAB (CIE1976) color system [4]. Each color can be mathematically disassembled into three tristimulus values $(\mathrm{X}, \mathrm{Y}, \mathrm{Z})$, which are imaginary colors representing the three fundamental colors (Blue, Green, Red). The three components of tristimulus can be defined as following:

$$
\begin{aligned}
& X=K \sum_{380}^{780} \varphi(\lambda) \bar{y}(\lambda) \Delta(\lambda) \\
& Y=K \sum_{380}^{780} \varphi(\lambda) \bar{y}(\lambda) \Delta(\lambda) \\
& Z=K \sum_{380}^{780} \varphi(\lambda) \bar{z}(\lambda) \Delta(\lambda)
\end{aligned}
$$

$\bar{x}(\lambda) \cdot \bar{y}(\lambda) \cdot \bar{z}(\lambda)$ are the CIE1931 standard observer color matching function. $\mathrm{K}$ is the normalized factor, $K=100 / \sum_{780}^{380} S(\lambda) \bar{y}(\lambda) \Delta(\lambda)^{[5-6] .} \varphi(\lambda)$ is defined as color stimulus function that represents the visible spectral distribution related to our eye's color response.

The color stimulus function $\varphi(\lambda)$ value is defined in three conditions: (1) $\varphi(\lambda)=S(\lambda)$, when the illuminant light source is directed into our eyes; (2) $\varphi(\lambda)=T(\lambda) S(\lambda)$, when the light is passed through a transparent object with transmittance $T(\lambda)$; (3) $\varphi(\lambda)=R(\lambda) S(\lambda)$, when the light is reflected from the surface of an object with reflectivity $R(\lambda)$.

CIE has established CIEXY chromaticity diagram representing the normalization of the three tristimulus values in a two-dimensional color space. The normalized sum of coordinates values always equal to $1(x+y+z=1)$.

$$
\begin{aligned}
& x=\frac{X}{X+Y+Z} \\
& y=\frac{Y}{X+Y+Z} \\
& z=\frac{Z}{X+Y+Z}
\end{aligned}
$$

However, in the CIEXYZ model, there are two problems with the expression of colors in terms of tristimulus values. First, the CIEXYZ is not perceptually uniform. Second, the two-dimensional color space doesn't include the lightness value associated with human psychophysical perception.
CIE has specified a three-dimensional color space, CIELAB, of which the $L^{*} a^{*} b^{*}$ space was experimentally designed to be perceptually linear [78]. The mathematical equation is defined as followed. The $\mathrm{L}^{*}$-axis is known as the lightness and extends from 0 (black) to 100 (white), and it's also called the achromatic scale. The other two coordinates $a^{*}$ and $b^{*}$ represent redness-greeness and yellowness- blueness respectively. It should be noted that the CIELAB is a non-linear transformation resulted from the three tristimulus values $\mathrm{X}, \mathrm{Y}, \mathrm{Z}$. CIELAB also includes the three major components, lightness, chroma, and hue value, respectively. The ratio of the $X / X_{n}, Y / Y_{n}$ and $Z / Z_{n}$ represent normalization, where the $X_{n}, Y_{n}$ and $Z_{n}$ are the tristimulus values of illuminant light source. Furthermore, CIE define two parameters simultaneously for calculated chroma and hue value, $\mathrm{a}^{*}$ and $\mathrm{b}^{*}$. When $\left(X / X_{n}\right)$ or $\left(Y / Y_{n}\right)$ or $\left(Z / Z_{n}\right) \geqq$ 0.008856 :

$$
\begin{gathered}
L^{*}=116\left(\frac{Y}{Y_{n}}\right)^{1 / 3}-16 \\
a^{*}=500\left[\left(\frac{X}{X_{n}}\right)^{1 / 3}-\left(\frac{Y}{Y_{n}}\right)^{1 / 3}\right] \\
b^{*}=200\left[\left(\frac{Y}{Y_{n}}\right)^{1 / 3}-\left(\frac{Z}{Z_{n}}\right)^{1 / 3}\right] \\
C^{*}{ }_{a b}=\left[\left(a^{*}\right)^{2}+\left(b^{*}\right)^{2}\right]^{1 / 2} \\
H_{a b}=\arctan \left(\frac{b^{*}}{a^{*}}\right)
\end{gathered}
$$

\section{MATERIALS AND METHODS}

In this study, we measured the reflected spectra of a set of tooth shade tabs (VITAPAN 3D-MASTER, Germany). It reflects the characteristics of multilayered, non-homogeneous and opaque properties. The reflected light from the central area of tooth were recorded to reduce the variations in all experiments. In addition, the colors of teeth were also affected by the specular reflection. Thus, we have conducted a series of experiments for choosing the optimal geometric angle and to reduce the specular light. We have achieved the best results at $\left(45^{\circ} \pm 15^{\circ}\right) /$ $0^{\circ}$ (light source/observer) arrangement (Figure.1).

Figure. 2 shows the schematic of the spectroscopic tooth colorimeter system, consisting of a $3600 \mathrm{mcd}\left(I_{V}\right)$ at $20 \mathrm{~mA}\left(\mathrm{I}_{\mathrm{F}}\right)$ high power white LED (NSPW300BS, NICHIA, JAPAN), a condensing lens, a miniature 
spectrometer (USB2000-UV-VIS, Ocean Optics, USA), two self-assembly plastic optical fibers and a set of teeth shade tabs.

The VITAPAN 3D-MASTER tooth shade tabs cover the entire color space of natural teeth and based on a systematically three-dimensional arrangement of teeth colors. There are three steps of standard selection rule recommended for visual color matching. First, choose the value (lightness) from five value groups that are closest to the one of the tooth to be compared. Second, select the chroma (saturation) that is the closest one. Finally, determine whether the natural tooth is more yellowish or more reddish by selecting the tooth on the right or left hand side.

The USB2000 spectrometer is a handheld spectrometer which can be plugged directly into the Universal Serial Bus (USB) interface. It was set-up at $25 \mu \mathrm{m}$ entrance slit, 600 lines $/ \mathrm{mm}$ grooves for $200 \sim 850 \mathrm{~nm}$ spectral range and 2048 pixel-element linear CCD-array detector; the optical resolution was $1.34 \mathrm{~nm}$ optical resolution. The optical resolution of the spectrometer is equal to the multiplication of dispersion and resolution: $650 \mathrm{~nm} / 2048$ pixels and slit width that corresponds to 4.2 pixels for $25 \mu \mathrm{m}$ slit size in this spectrometer [9].

The distributions of most teeth shade lay on the whiteness and yellowness region. Therefore, we have presented a new expression, WYI (whitenessyellowness index) value, by incorporating the two tooth color indices: the YI (yellowness index; ASTM D1925 formula) value and WI (whiteness index; CIE' 82 , Berger) value. The WYI can be easily used in clinical tooth cosmetic treatments (especially in tooth whitening and prosthesis), it is describes as followed:

$$
\begin{gathered}
Y . I .=\frac{128 X-106 Z}{Y} \\
W . I=Y+a\left(x_{0}-x\right)+b\left(y_{0}-y\right) \\
W . Y . I .=\left(\frac{W_{\max }-W^{\prime}}{W_{\max }-W_{\min }}\right)+\left(\frac{Y^{\prime}-Y_{\min }}{Y_{\max }-Y_{\min }}\right)
\end{gathered}
$$

Where $\mathrm{X}, \mathrm{Y}$ and $\mathrm{Z}$ are represented the three tristimulus values; $a=800$ and $b=1700$ in neutral hue. $\mathrm{x}_{0}, \mathrm{y}_{0}$ are the coordinate values of illuminant light source in CIEXYZ model, and $\mathrm{x}, \mathrm{y}$ are represented the values of tooth shade tabs in the same model; $\mathrm{W}_{\max }$, $\mathrm{W}_{\min }, \mathrm{Y}_{\max }, \mathrm{Y}_{\min }$ are represented the maximum and minimum values in the formula W.I. and Y.I. respectively. In the VITAPAN 3D-MASTER tooth shade tabs, $W^{\prime}$ and $Y^{\prime}$ are the value while we change the tooth samples.

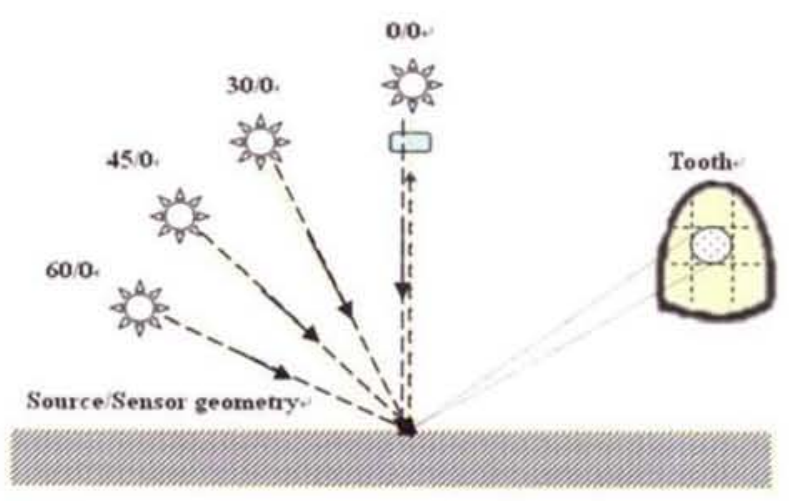

Fig.1 The Geometric angles of reflectance color of teeth samples.

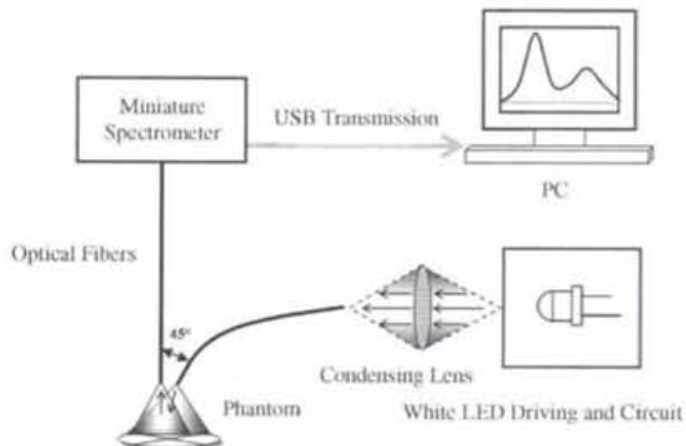

Fig.2 Schematic of Fiber optics spectroscopic tooth colorimeter

\section{RESULTS AND DISCUSSIONS}

Figure.3 (a) shows the back scattered spectra of the VITAPAN 3D-MASTER tooth shade samples. The area normalization of the spectra was shown in Figure.3 (b). The normalized spectra can be easily characterized by the peak position. The intensity of the first primary peak, at $467 \mathrm{~nm}$, is different that represents the lightness values of different teeth are varying. The second primary peak, at $550 \mathrm{~nm}-560 \mathrm{~nm}$, represents the hue value shifts towards reddish colors. Figure. 3 (c) represents the zoom of the second normalized spectra peak.

Figure.4 shows the results in CIEXYZ model, the coordinate $(x, y)=(0.33,0.33)$ is defined as the CIE standard illuminant $\mathrm{E}$, which is an achromatic point. The $(x, y)$ value of the white LED is at $(0.33,0.34)$, which is almost overlap to the CIE standard illuminant E. We have marked $\lceil\times\rfloor$ to indicate the position of 26 tooth samples.

Figure. 5 shows the results in the chromaticity of the CIELAB model, we demonstrated that teeth 


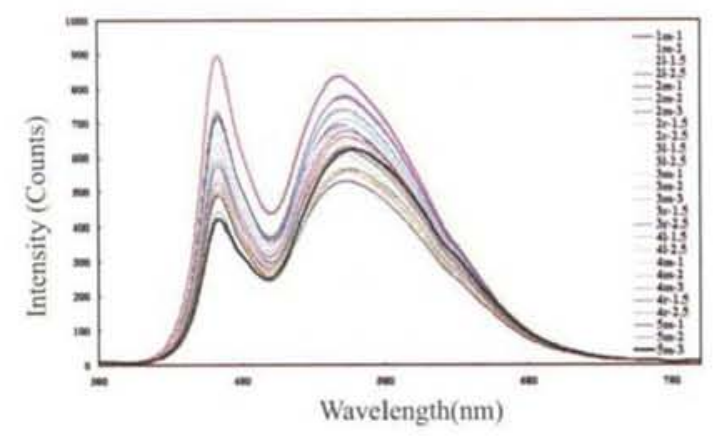

Fig.3 (a) The Back scattered spectra from shade tabs of VITAPAN 3D-MASTER

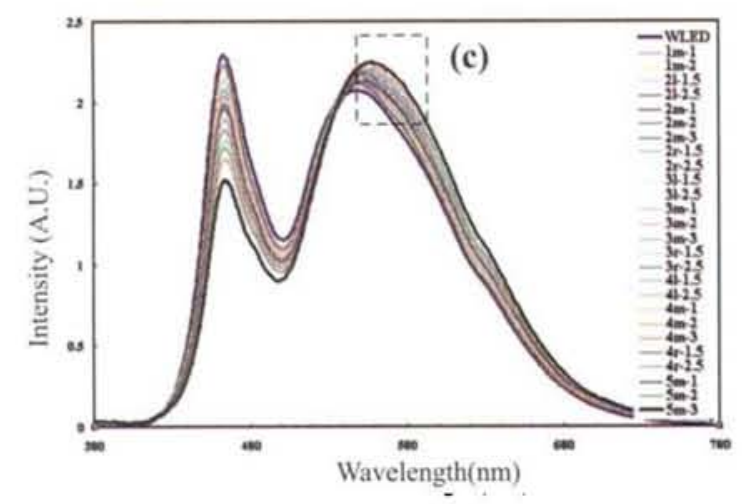

Fig.3 (b) Area normalization of back scattered spectra.

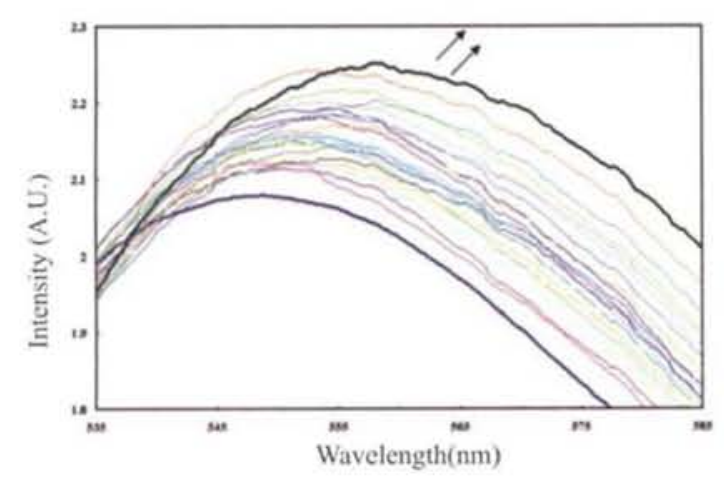

Fig.3 (c) The zoom of the second area normalized spectra.

phantoms were in the red-yellow region (positive $\mathrm{a}^{*}$ and positive $\mathrm{b}^{*}$ ), and it is also conformed to the visualization of human perception. The tooth shade tabs is more yellowish while the lightness value decreases.

Figure.6 shows the lightness value of object in the CIELAB model which is opposite to chroma value.

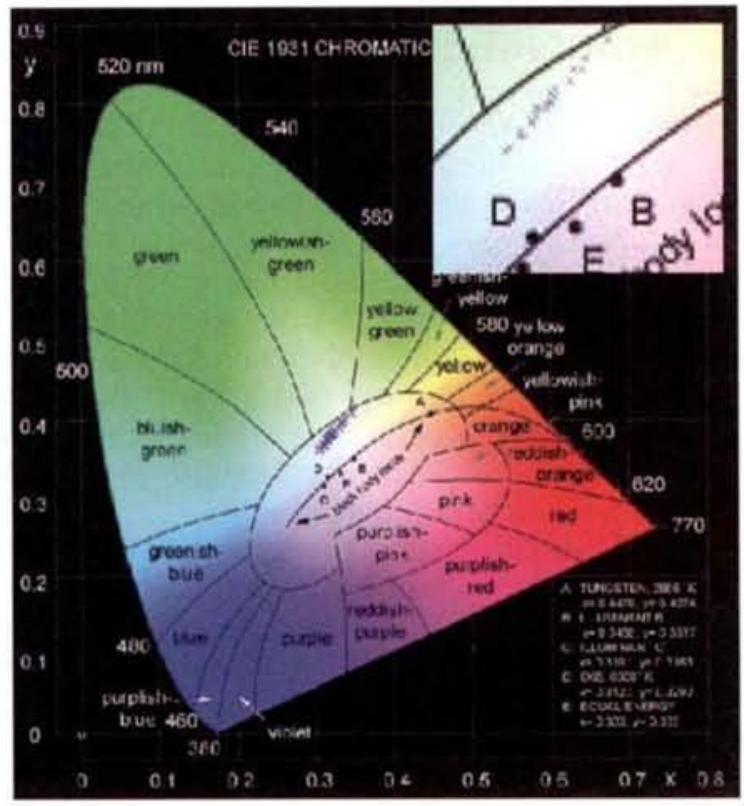

Fig.4 The CIEXYZ (CIE1931) chromaticity diagram of the 26 teeth samples, $\lceil\times\rfloor$, and the zoom of the 26 shade tabs

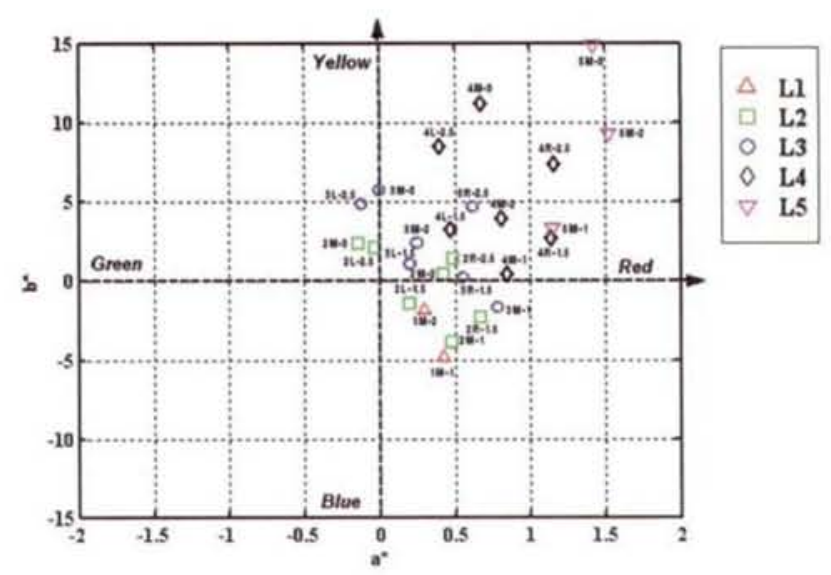

Fig.5 Teeth samples distributions on CIELAB (CIE 1976) color space, $a^{*}$ axis represents the Green-Red value; $b^{*}$ axis represents the BlueYellow value.

This observation is similar in our sensation, and also same as the principle of colorimetry. While the lightness values are decreased from L1 to L5, the chroma value of teeth samples is increased.

We combined the normalized WI and YI indices to form a simplified color coding representation WYI model (Figure.7). The logical configuration is a 
quantitative measurement showing the color information and tooth matching results.

In addition, we combined the WI and YI values and observed the linear relationship between WI and YI (Figure.8). The results shows that the design of the tooth shade tabs has took into account the linearity arrangements and achievements of the well controlled fabrication process. The combination of WI and YI to be determined the teeth color in 2D spectra. Hence, we can use the 3D tooth shade tabs for color discrimination of the teeth.

\section{CONCLUSION}

Tooth colorimeter is an important and quantitative instrument in clinical dental operations. In this tooth

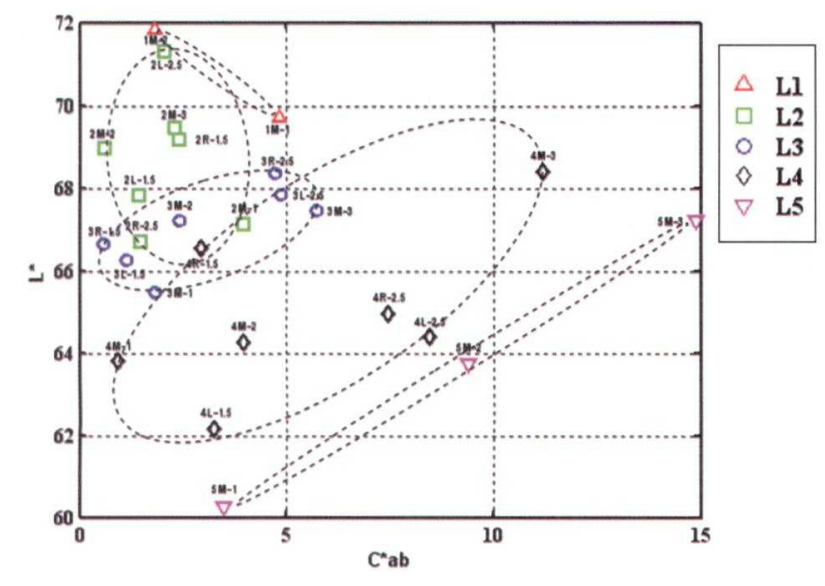

Fig.6 Lightness $\left(L^{*}\right)$ and Chroma $\left(C^{*} a b\right)$ variations of CIELAB color space, each $L^{*}$ group is encircled by dash line

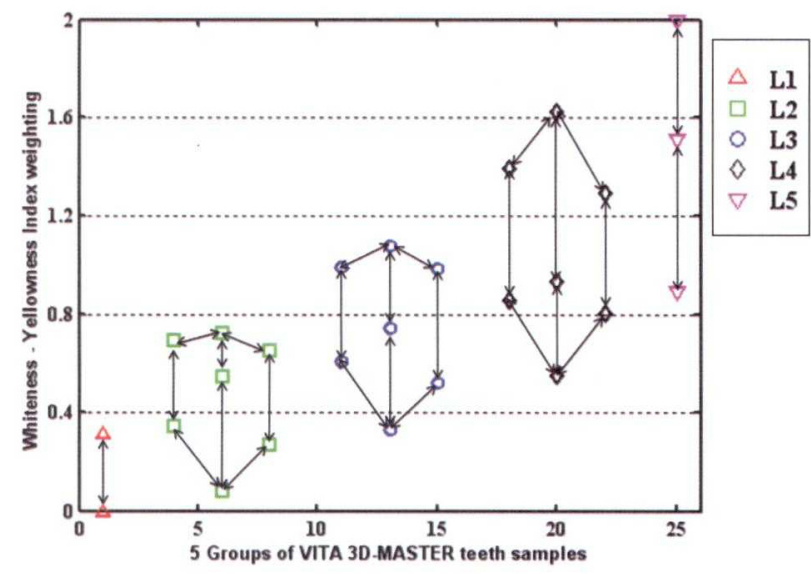

Fig.7 The new whiteness - yellowness index (W.Y.I.) representation

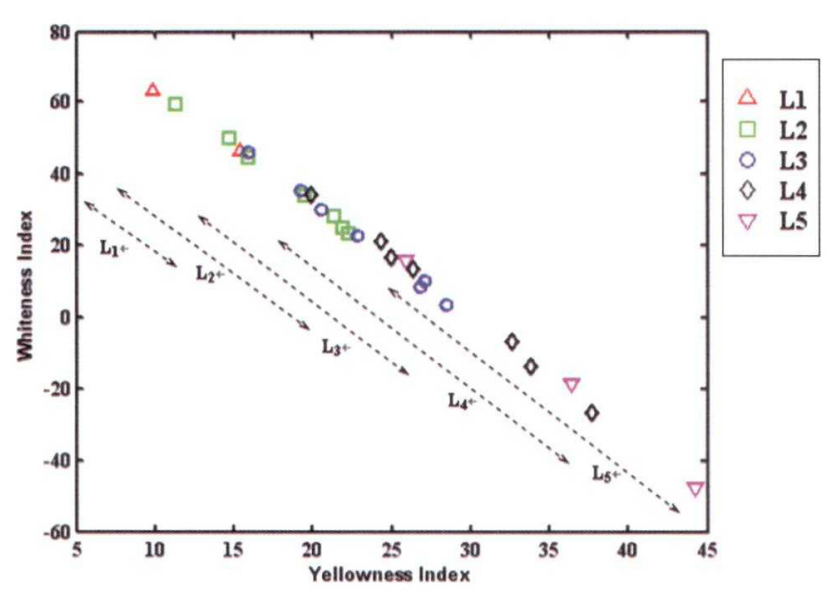

Fig.8 The linear relationship between the whiteness index (W.I.) and yellowness index (Y.I.)

colorimetric system, we have used optical fibers, miniature spectrometer, and white LED to develop the system. We have developed the WYI index and implemented in clinical tooth shade samples. We have successfully analyzed the color difference between shade phantoms by using the developed colorimeter. In this research, a simple, fast and precise fiber opticsbased spectroscopic tooth colorimeter was developed and demonstrated to be an efficient and accurate color analyzer. In the near future, we would explore the color matching by using the colorful image sensing approaches for providing a more sensitive color resolution [10-12].

\section{ACKNOWLEDGEMENT}

This research was supported by the National Science Council, Taiwan, under grant NSC 91-2622-E010-003-CC3.

\section{REFERENCE}

1. Lichter JA, Solomowitz BH, Sher M: Shade selection. Communicating with the laboratory technician. NY State Dent J 2000; 66(5): 42-6.

2. Pensler AV: Shade selection problems and solutions. Compend contin Educ Dent 1998; 19(4): 387-90m 392-4.

3. Sagara. J.: Shade matching for today's dentistry. Dental Economics 2002; 62-68.

4. Gonzalez, Rafael C., Richard E. Woods: Digital Image Processing, Prentice Hall, 2001.

5. Mark Shaw, Mark Fairchild: Spectral Power Distributions for the CIE Stimuli. Color Res Appl $2001 ; 26: 478-482$ 
6. Mark Shaw, Mark Fairchild: Evaluating the 1931 CIE Color Matching Functions. Color Res Appl 2002; 27:316-329.

7. CIE: Uniform Color Spaces-Color Difference Equations - Psychometric Color Terms, Commission Internationale de L'Eclairage, Publication No. 15, Supplement No. 2, Paris, 1978.

8. MacAdam, D.L.:Visual Sensitivities to Color Differences in Daylight. J. Opt. Soc. Am., 1942; $32: 247-274$.
9. http://oceanopticsbv.com/technical/opticalresolution.asp 10. Maryann Lehmann: US patent 6358 047, May 19, 2002.

11. Gregg A. Wagner, Boulder, Gary Emerson, Golden: US patent 6157 454, Dec. 5, 2000.

12. Morovic J., Morovic P.: Determining colour gamuts of digital cameras and scanners. Color Res Appl 2003; 28:59-68. 